Multimed Tools Appl

DOI $10.1007 / \mathrm{s} 11042-015-2976-8$

\title{
A Web video retrieval method using hierarchical structure of Web video groups
}

\author{
Ryosuke Harakawa - Takahiro Ogawa • \\ Miki Haseyama
}

Received: 17 February 2015 / Revised: 10 September 2015 / Accepted: 29 September 2015

\begin{abstract}
In this paper, we propose a Web video retrieval method that uses hierarchical structure of Web video groups. Existing retrieval systems require users to input suitable queries that identify the desired contents in order to accurately retrieve Web videos; however, the proposed method enables retrieval of the desired Web videos even if users cannot input the suitable queries. Specifically, we first select representative Web videos from a target video dataset by using link relationships between Web videos obtained via metadata "related videos" and heterogeneous video features. Furthermore, by using the representative Web videos, we construct a network whose nodes and edges respectively correspond to Web videos and links between these Web videos. Then Web video groups, i.e., Web video sets with similar topics are hierarchically extracted based on strongly connected components, edge betweenness and modularity. By exhibiting the obtained hierarchical structure of Web video groups, users can easily grasp the overview of many Web videos. Consequently, even if users cannot write suitable queries that identify the desired contents, it becomes feasible to accurately retrieve the desired Web videos by selecting Web video groups according to the hierarchical structure. Experimental results on actual Web videos verify the effectiveness of our method.
\end{abstract}

Keywords Web video retrieval - Web video group · hierarchical structure · strongly connected component $\cdot$ edge betweenness $\cdot$ modularity

\section{Introduction}

According to IDC reports [9], the digital universe in 2005 was 130 exabytes and will be 300 times larger (40 zettabytes) in 2020. Also, most of the data in the digital universe are unstructured data including Web videos ${ }^{1}$. Therefore, more and

R. Harakawa · T. Ogawa · M. Haseyama

Graduate School of Information Science and Technology,

Hokkaido University, Sapporo, Japan

Tel.: +81-11-706-6078

Fax: +81-11-706-7369

E-mail: \{harakawa, ogawa\}@lmd.ist.hokudai.ac.jp, miki@ist.hokudai.ac.jp

1 In this paper, we call video materials on the Web "Web videos". 
more users are retrieving Web videos to access the desired contents, i.e., contents with topics that users try to find. Here, topics contained in the desired contents belong to various hierarchical levels according to each user. Given topics related to "Apple Inc." as an example, a user may desire contents with "iPhone of Apple Inc."; meanwhile, another user may desire contents with "iPhone, iPad and their related devices".

When users retrieve Web videos using existing retrieval systems such as YouTube ${ }^{2}$, they input queries into the systems in order to narrow down the enormous amount of data to a suitable size [13]. However, it is difficult for users to write suitable queries that accurately identify the desired contents. Below, we show two examples, i.e., a case when the enormous amount of data cannot be sufficiently narrowed down and another when the data is narrowed down too much. First, assume that a user desires contents with "iPhone of Apple Inc." If this user writes a query "Apple", the data cannot be narrowed down sufficiently. Second, assume that another user desires contents with "iPhone, iPad and their related devices". If this user writes a query "iPhone" or "iPad", the data is narrowed down too much. In this way, it is not necessarily easy for users to write suitable queries. In such a case, it becomes difficult for users to find the desired Web videos from the retrieval results, which are narrowed down by unsuitable queries [14].

To solve this problem, methods based on exhibition of similar Web video sets can be useful $[10,16,17,31]$. By using these methods, users can retrieve the desired Web videos since they can effectively browse the retrieval results via the similar Web video sets. In an article [31], a clustering method based on visual features for near-duplicate video retrieval was proposed. A work [10] proposed a clustering method that uses co-watching of Web videos and textual features of Web videos, i.e., features of text attached to Web videos such as title and description. In a study [17], a clustering method of retrieval results based on visual and textual features of Web videos was proposed. Also, a paper [16] proposed a retrieval method that provides similar Web video sets on the basis of heterogeneous video features, i.e., visual, audio and textual features and link relationships between Web videos. However, these clustering-based methods cannot provide results including topics at various hierarchical levels. Remember the above example, i.e., a user that desires contents with "iPhone, iPad and their related devices". Here, if the obtained clusters include topics at the upper level, e.g., "Apple", a user still needs to find the desired contents from the clusters. On the other hand, if the obtained clusters include topics at the lower level, e.g., "iPhone", the desired contents may not exist in the clusters.

To overcome this difficulty, hierarchical clustering-based retrieval methods that can provide results including topics at various hierarchical levels are necessary [7, $23,28,30]$. If hierarchical structure of Web video sets, which includes many topics, is provided, the hierarchical structure can navigate users to the desired contents (see Fig. 1). A work [28] proposed a Web video retrieval method based on exhibition of hierarchical topic structure obtained by using textual analysis and WordNet [20]. In a paper [23], a hierarchical scheme that first clusters video shots into ones with similar color and then clusters video shots into ones with similar motion to retrieve videos about sports games. The method in [7] constructs the hierarchical tree structure of semantic-sensitive video classifier for indexing and

2 http://www.youtube.com 


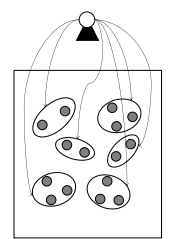

(a)

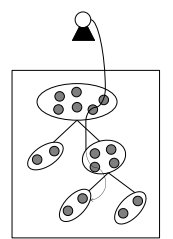

(b)

Fig. 1 Schemes to provide Web video sets. (a) Non-hierarchical scheme: If topics contained in the Web video sets and those contained in the desired contents do not belong to the same hierarchical levels, retrieval of the desired contents becomes difficult. (b) Hierarchical scheme: The hierarchical structure can navigate users to the desired contents.

effective video access. The method in [30] performs clustering of the similar video shots in a hierarchical fashion for browsing. Although these methods can overcome the above difficulty, they utilize only a single modality, i.e., textual features or visual features. Therefore, these conventional methods have the limitations in their retrieval performance due to the lack of useful information obtained from the other modalities.

Hence, the above conventional methods have the following two problems:

(Problem-i) The retrieval performance may be limited since only a single modality is used. This is the problem in the earlier works $[7,23,28,30]$.

(Problem-ii) Web video retrieval is performed without the use of the hierarchical structure of similar Web video sets. This is the problem in the conventional studies $[10,16,17,31]$.

Therefore, in this paper, we propose a Web video retrieval method that uses hierarchical structure of Web video groups. In this paper, Web video groups are defined as Web video sets with similar topics. Also, their hierarchical structure denotes the property of Web video groups being divided into the sub-groups in this paper. Our contribution of this paper is the followings:

(Contribution-i) Hierarchical structure of Web video groups is estimated by using link relationships obtained via metadata "related videos" and heterogeneous video features, i.e., visual, audio and textual features.

(Contribution-ii) Web video retrieval is performed by exhibiting the hierarchical structure to users.

In (Contribution-i), we analyze a network constructed by using link relationships and heterogeneous video features on the basis of strongly connected components (SCCs) [5], edge betweenness [22] and modularity [2] to successfully estimate the hierarchical structure. In (Contribution-ii), even if users cannot write suitable queries, the hierarchical structure can navigate users to the desired contents. Finally, we note that this paper is an extended version of $[12]^{3}$.

This paper is organized as follows. In Sec. 2, an outline of the proposed Web video retrieval method is presented. In Sec. 3, a method for selecting representative Web videos based on Web video features is described. A method for extracting the hierarchical structure of Web video groups is explained in Sec. 4. A Web

\footnotetext{
3 In this paper, the method in [12] is improved by automatically deciding the number of hierarchies exhibited to users on the basis of modularity.
} 


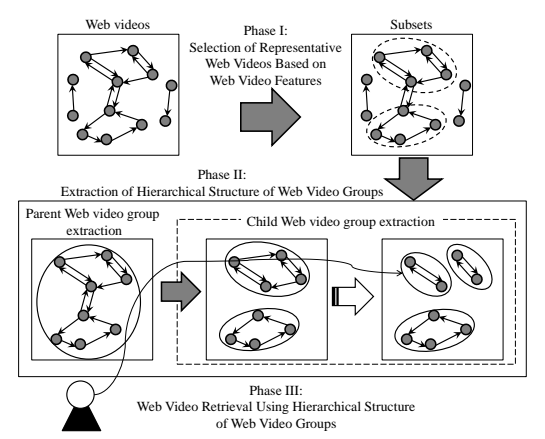

Fig. 2 Outline of the proposed Web video retrieval method using hierarchical structure of Web video groups.

video retrieval method that uses the hierarchical structure is presented in Sec. 5 . In Sec. 6, experimental results on actual Web videos are shown to verify the effectiveness of our method. Limitation and future work of this paper is described in Sec. 7. Concluding remarks are given in Sec. 8.

\section{Outline of Web Video Retrieval Using Hierarchical Structure of Web video groups}

In this section, an outline of the proposed Web video retrieval method using hierarchical structure of Web video groups is presented. Figure 2 shows an outline of the proposed method. As shown in the figure, our method consists of three phases. An overview of them is shown below.

Phase I: Selection of Representative Web Videos Based on Web Video Features This phase is useful for screening irrelevant contents from many Web videos. As a result, this phase enables both reduction of computational cost in Phase II and accurate retrieval of users' desired contents in Phase III.

Phase II: Extraction of Hierarchical Structure of Web Video Groups

This phase aims to provide hierarchical structure of Web video groups related to a given query. By providing the hierarchical structure, it becomes feasible for users to easily find the desired contents.

Phase III: Web Video Retrieval Using Hierarchical Structure of Web Video Groups This phase enables to rank Web videos in each Web video group. Thus, users can retrieve Web videos in the descending order of the centrality in the selected Web video group.

The details of Phases I, II and III are presented in Secs. 3, 4 and 5, respectively.

\section{Selection of Representative Web Videos Based on Web Video Features (Phase I)}

In this section, a method for selecting representative Web videos based on Web video features is explained. 


\subsection{Definition of Web Video Features}

In this subsection, we explain the definition of Web video features used in this paper. In this paper, Web video features are calculated from Web videos $f_{i}(i=$ $1,2, \cdots, N ; N$ being the number of Web videos) according to a paper [16]. First, we apply the shot segmentation method [21] to each Web video $f_{i}$, and obtain several shots $s_{i}^{q_{i}}\left(q_{i}=1,2, \cdots, M_{i} ; M_{i}\right.$ being the number of shots within $\left.f_{i}\right)$. Then a visual feature vector $\boldsymbol{v}_{i}^{q_{i}}$, an audio feature vector $\boldsymbol{a}_{i}^{q_{i}}$ and a textual feature vector $\boldsymbol{t}_{i}^{q_{i}}$ are calculated from each shot $s_{i}^{q_{i}}$ as follows.

Visual Feature Vector ( $p$ dimensions)

We calculate the HSV color histogram with $p$ bins every $P_{v}$ frames of a Web video $f_{i}$ to obtain its vector. For the frames in each shot $s_{i}^{q_{i}}$, the vector median [3] is calculated to obtain $\boldsymbol{v}_{i}^{q_{i}}\left(=\left[v_{i}^{q_{i}}(1), v_{i}^{q_{i}}(2), \cdots, v_{i}^{q_{i}}(p)\right]^{\mathrm{T}}\right)$. As a result, we obtain $M_{i}$ visual feature vectors $\boldsymbol{v}_{i}^{q_{i}}\left(q_{i}=1,2, \cdots, M_{i}\right)$ from each Web video $f_{i}$.

Audio Feature Vector (22 dimensions)

Based on a study [25], we calculate 22 dimensional audio feature vectors $\boldsymbol{a}_{i}^{q_{i}}$ that are useful for audio signal classification for each shot $s_{i}^{q_{i}}$. For more details, refer to a paper [16].

Textual Feature Vector ( $r$ dimensions)

Let $K$ be the total number of keywords that appear in text attached to Web videos $f_{i}(i=1,2, \cdots, N)$. We apply TF-IDF [29] to the $K$ keywords, and calculate the vector $\boldsymbol{\eta}_{i}\left(=\left[\eta_{i}(1), \eta_{i}(2), \cdots, \eta_{i}(K)\right]^{\mathrm{T}}\right)$ by aligning the obtained weights. Furthermore, we apply principal component analysis (PCA) to $\boldsymbol{\eta}_{i}$ $(i=1,2, \cdots, N)$ to reduce their dimensions, and obtain new $r$-dimensional vectors $\boldsymbol{t}_{i}$. Thereby, for each shot $s_{i}^{q_{i}}$ in Web video $f_{i}$, we obtain $M_{i}$ textual feature vectors $\boldsymbol{t}_{i}^{q_{i}}\left(=\boldsymbol{t}_{i}\right)\left(q_{i}=1,2, \cdots, M_{i}\right)$.

Thus, from each shot $s_{i}^{q_{i}}$ in the Web video $f_{i}$, the visual feature vector $\boldsymbol{v}_{i}^{q_{i}}$, the audio feature vector $\boldsymbol{a}_{i}^{q_{i}}$ and the textual feature vector $\boldsymbol{t}_{i}^{q_{i}}$ are calculated.

\subsection{Selection of Representative Web Videos}

In this subsection, a method for selecting representative Web videos by using the three kinds of features and link relationships between Web videos is explained. First, in order to obtain variates that can be compared between the different kinds of features, canonical correlation analysis (CCA) [24] is applied to $\boldsymbol{v}_{i}^{q_{i}}, \boldsymbol{a}_{i}^{q_{i}}$ and $\boldsymbol{t}_{i}^{q_{i}}$. Then we obtain $\boldsymbol{\zeta}_{\boldsymbol{v}_{i}^{q_{i}}}^{l}, \boldsymbol{\zeta}_{\boldsymbol{a}_{i}^{q_{i}}}^{l}$ and $\boldsymbol{\zeta}_{\boldsymbol{t}_{i}^{q_{i}}}^{l}$, which are the projection results of $\boldsymbol{v}_{i}^{q_{i}}, \boldsymbol{a}_{i}^{q_{i}}$ and $\boldsymbol{t}_{i}^{q_{i}}$ into the latent space of $l(\in\{v, a, t\})$, where $v, a$ and $t$ correspond to visual, audio and textual features, respectively. In the experiments shown later, the three kinds of feature vectors were projected into the latent space of textual features by setting $l=t$. For more details of this calculation, refer to a paper [16].

Next, by using $\boldsymbol{\zeta}_{\boldsymbol{v}_{i}^{q_{i}}}^{l}, \boldsymbol{\zeta}_{\boldsymbol{a}_{i}^{q_{i}}}^{l}$ and $\boldsymbol{\zeta}_{\boldsymbol{t}_{i}^{q_{i}}}^{l}$, we calculate similarities $S(i, j)$ between Web videos $f_{i}$ and $f_{j}$ by the following equations:

$$
\begin{array}{r}
S(i, j)=\max _{q_{i}, q_{j}}\left|\frac{\left(\boldsymbol{\xi}_{i}^{q_{i}}\right)^{\mathrm{T}} \boldsymbol{\xi}_{j}^{q_{j}}}{\left\|\boldsymbol{\xi}_{i}^{q_{i}}\right\|\left\|\boldsymbol{\xi}_{j}^{q_{j}}\right\|}\right| \\
\boldsymbol{\xi}_{i}^{q_{i}}=\left[\left(\boldsymbol{\zeta}_{\boldsymbol{v}_{i}^{q_{i}}}^{l}\right)^{\mathrm{T}},\left(\boldsymbol{\zeta}_{\boldsymbol{a}_{i}^{q_{i}}}^{l}\right)^{\mathrm{T}},\left(\boldsymbol{\zeta}_{\boldsymbol{t}_{i}^{q_{i}}}^{l}\right)^{\mathrm{T}}\right]^{\mathrm{T}} .
\end{array}
$$


In this way, we calculate the similarities between Web videos by integrating the heterogeneous video features on the basis of CCA.

Furthermore, we weight the adjacency matrix that represents link relationships between Web videos and calculate the weighted adjacency matrix $\boldsymbol{L}_{w}$ whose $(i, j)$ th element $L_{w}(i, j)$ is as follows:

$$
L_{w}(i, j)=\left\{\begin{array}{ll}
S(i, j) & \text { if } f_{i} \text { links to } f_{j} \\
0 & \text { otherwise }
\end{array},\right.
$$

where $i=1,2, \cdots, N$ and $j=1,2, \cdots, N$. In the above equation, we build link relationships between Web videos based on metadata "related videos". In particular, we consider that a Web video $f_{i}$ links to a Web video $f_{j}$ if "related videos" of $f_{i}$ include $f_{j}$. Since the metadata "related videos" are useful for obtaining Web videos related to each other [6], we introduce them into our method.

Moreover, we calculate $N_{g}$ eigenvectors of $\boldsymbol{L}_{w}^{\mathrm{T}} \boldsymbol{L}_{w}$, where $N_{g}$ is the number of the calculated eigenvectors. Each element of the eigenvectors of $\boldsymbol{L}_{w}^{\mathrm{T}} \boldsymbol{L}_{w}$ represents the attribution degree of each Web video to sets of Web videos with similar contents [19]. Next, we select $N_{m}$ Web videos in descending order of the element values of each eigenvector, and represent these Web videos as $C_{q}\left(q=1,2, \cdots, N_{g}\right)$. In this paper, Web videos contained in $C_{q}$ are called a "subset". By extracting the subset, we can obtain representative Web videos in the network of link relationships between Web videos since Web videos that densely link to each other can be extracted [19]. Thus, we can filter out irrelevant Web videos from many ones.

\section{Extraction of Hierarchical Structure of Web Video Groups (Phase II)}

In this section, a method for extracting hierarchical structure of Web video groups is explained. In our method, we summarize Web videos related to each other as Web video groups based on SCCs [5] and extract their hierarchical structure by using edge betweenness [22] and modularity [2]. Since SCCs are useful to find Web videos related to each other in the network of link relationships between Web videos, we introduce this measure to obtain "parent Web video groups". Moreover, a study [22] reports that the combination use of edge betweenness and modularity enables extraction of the significant hierarchical structure. Therefore, we adopt these two measures to extract the hierarchical structure as in [22]. Specifically, we extract the hierarchical structure by repeatedly dividing parent Web video groups into their "child Web video groups" 4 on the basis of edge betweenness and modularity. In this paper, we collectively call parent Web video groups and child Web video groups "Web video groups". Next, we explain the details of this scheme.

\subsection{Construction of Parent Web Video Groups}

First, from Web videos that belong to the subset $C_{q}\left(q=1,2, \cdots, N_{g}\right)$, a weighted and directed network $G=(V, E)$, whose nodes and edges are respectively Web

\footnotetext{
4 When a child Web video group is divided into its child Web video groups, this child Web video group is considered to be the parent Web video group of its child Web video groups.
} 


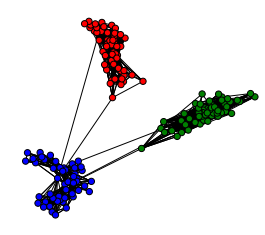

(a) $N_{\text {cut }}=0$

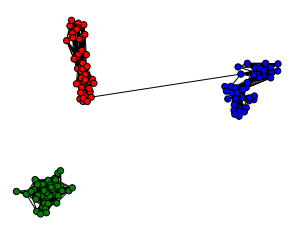

(b) $N_{\text {cut }}=15$

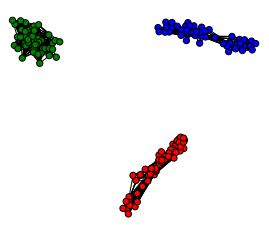

(c) $N_{\text {cut }}=30$

Fig. 3 Simple example of Web video groups. (a) A parent Web video group is formed. (b) Two child Web video groups are formed by dividing their parent Web video group. (c) Three child Web video groups are generated from their parent Web video group.

videos and links between these videos, is constructed. When a Web video $f_{i} \in V$ links to $f_{j} \in V$, the edge weight $e_{i j}$ from $f_{i}$ to $f_{j}$ is defined as follows:

$$
e_{i j}=L_{w}(i, j) \text {. }
$$

Thus, the edge weight represents the similarity between Web videos. When $f_{i} \in V$ does not link to $f_{j} \in V$, we do not define the edge $e_{i j}$. In our method, parent Web video groups are obtained by extracting SCCs. SCCs are sub-networks that have a directed path between any two nodes in a directed network. A method for extracting SCCs from a network is called SCC decomposition. In a study [5], related Web pages are grouped on the basis of SCC decomposition. In the proposed method, by applying SCC decomposition to $G$, all SCCs are extracted. Then these obtained components are defined as parent Web video groups. By extracting parent Web video groups, we can obtain Web video sets with similar topics.

\subsection{Extraction of Child Web Video Groups}

Next, we extract child Web video groups by repeatedly dividing the parent Web video groups on the basis of edge betweenness. Edge betweenness is used for detecting communities in a network [22]. Edge betweenness is defined by the number of the shortest paths that go through a target edge, and it is useful for detecting influential edges in a network. Let $c_{B}(e)$ be edge betweenness of an edge $e \in E$, and $c_{B}(e)$ is defined as follows:

$$
c_{B}(e)=\sum_{s, t \in V} \frac{\sigma(s, t \mid e)}{\sigma(s, t)}, \quad e \in E,
$$

where $\sigma(s, t \mid e)$ is the number of shortest paths from node $s$ to node $t$ that pass an edge $e$, and $\sigma(s, t)$ is the total number of shortest paths from node $s$ to node $t$. In our method, by iteratively removing edges with the largest edge betweenness in $G$ and re-applying SCC decomposition to $G$, we re-extract SCCs. In this paper, we denote the number of these iterations by $N_{c u t}$. Here, child Web video groups are extracted when a single SCC is divided into several SCCs (see Fig. 3). By repeatedly dividing the parent Web video groups into their child Web video groups, the hierarchical structure of the Web video groups is obtained. Note that each child 
Web video group becomes equivalent to each Web video when all edges in $G$ are removed. Since it is not effective for Web video retrieval to provide all hierarchies to users, we have to decide when our method stops dividing parent Web video groups into their child Web video groups.

\subsection{Introduction of Modularity}

To solve the above problem, we use a quality function called modularity, which evaluates the results of division of communities in a network. Note that the node set $V$ of $G=(V, E)$ does not contain all Web videos $f_{i}(i=1,2, \cdots, N)$ since $G$ is constructed by Web videos that belong to the extracted subset $C_{q}(q=$ $\left.1,2, \cdots, N_{g}\right)$. Therefore, in order to calculate modularity of $G$, we define the matrix $\boldsymbol{M}=\left(m_{i j}\right)$ as follows:

$$
m_{i j}=\left\{\begin{array}{lc}
L_{w}(i, j) & \text { if } f_{i} \in V \text { and } f_{j} \in V \\
0 & \text { otherwise }
\end{array}\right.
$$

where $i=1,2, \cdots, N$ and $j=1,2, \cdots, N$. Let $Q_{N_{c u t}}$ be modularity of $G$ where the number of cut edges is $N_{c u t}$, and $Q_{N_{c u t}}$ is defined as follows [2]:

$$
Q_{N_{c u t}}=\frac{1}{2 m} \sum_{i=1}^{N} \sum_{j=1}^{N}\left(m_{i j}-\frac{m_{i}^{\text {out }} m_{j}^{\text {in }}}{2 m}\right) \delta(i, j),
$$

where $2 m=\sum_{i=1}^{N} \sum_{j=1}^{N} m_{i j}, m_{i}^{\text {out }}=\sum_{j=1}^{N} m_{i j}, m_{j}^{i n}=\sum_{i=1}^{N} m_{i j}$ and $\delta(i, j)$ is 1 if $f_{i}$ and $f_{j}$ belong to the same Web video group and 0 otherwise. When significant community structure is revealed, $Q_{N_{c u t}}$ becomes close to 1, i.e., the maximum value of $Q_{N_{\text {cut }}}$. On the other hand, a network is divided into communities randomly, and $Q_{N_{c u t}}$ becomes close to 0 . In this paper, the $N_{c u t}$ value when $Q_{N_{\text {cut }}}$ is maximum is denoted by $N_{c u t}^{o p t}$. The proposed method uses the Web video groups, where $N_{\text {cut }}$ is from 0 to $N_{c u t}^{o p t}$ for Web video retrieval.

In this way, we hierarchically extract Web video groups based on SCCs, edge betweenness and modularity. Consequently, the entire contents of many Web videos can be easily grasped by obtaining the hierarchical structure of Web video groups. Algorithm 1 shows the specific procedures for extracting the hierarchical structure.

\section{Web Video Retrieval Using Hierarchical Structure of Web Video Groups (Phase III)}

In this section, a Web video retrieval method using the hierarchical structure of Web video groups is presented. We obtain Web video groups where $N_{\text {cut }}$ is from 0 to $N_{c u t}^{o p t}$. In this paper, these Web video groups are represented as $\operatorname{Com}_{N_{\text {cut }}}^{q}\left(N_{c u t}=\right.$ $0,1, \cdots, N_{c u t}^{\text {opt }}, q=1,2, \cdots, T_{N_{\text {cut }}} ; T_{N_{\text {cut }}}$ being the number of Web video groups in which the number of cut edges is $\left.N_{\text {cut }}\right)$. First, each Web video $f_{i}(i \in\{1,2, \cdots, N\})$ 


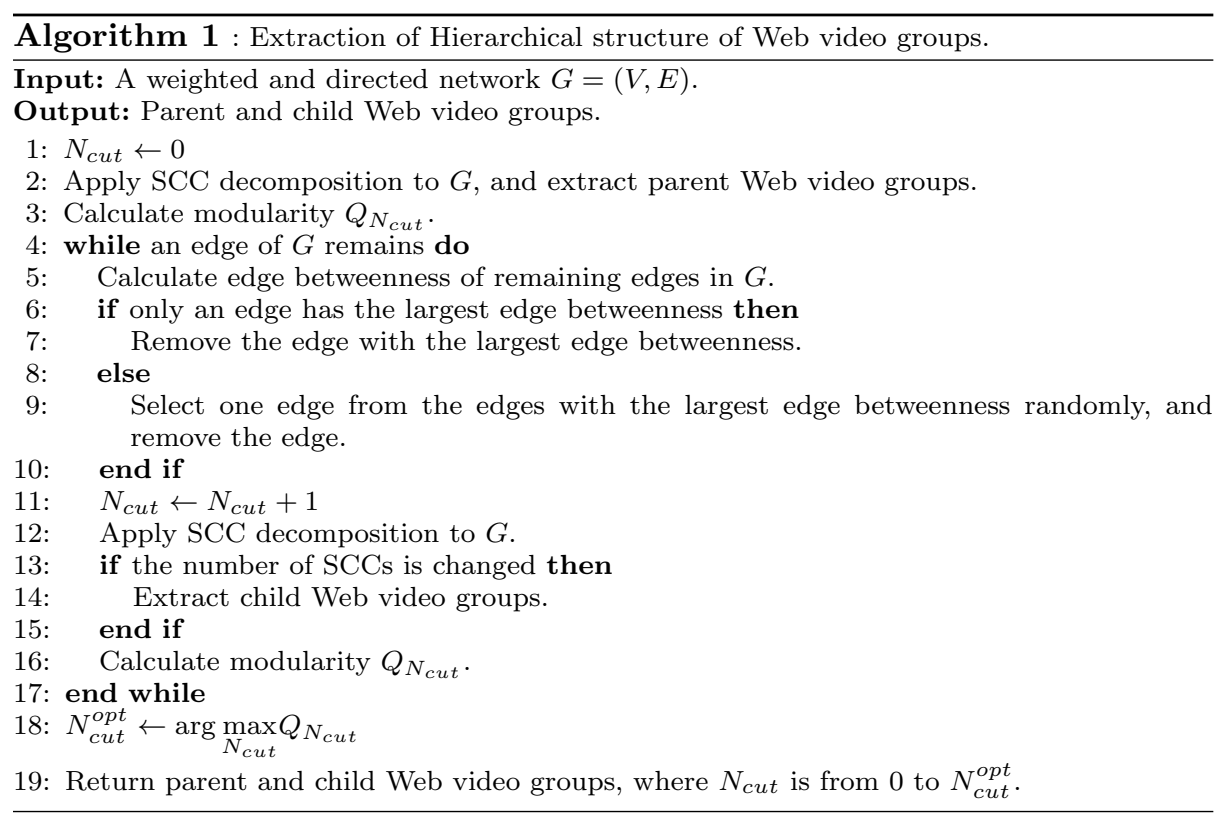

that belongs to the Web video group $\operatorname{Com}_{N_{c u t}}^{q}$ is ranked in descending order of the following criterion $R_{N_{c u t}}^{q}(i)$ :

$$
R_{N_{c u t}}^{q}(i)=\sum_{j=1}^{N} m_{j i} \delta(i, j),
$$

where $\delta(i, j)$ is 1 if $f_{i}$ and $f_{j}$ belong to the same Web video group and 0 otherwise. Hence, Web videos belonging to each Web video group are ranked on the basis of weighted links from other Web videos in the same Web video group.

Next, we exhibit the Web video groups $\operatorname{Com}_{N_{c u t}}^{q}$, where $N_{c u t}$ is from 0 to $N_{c u t}^{\text {opt }}$. Here, the smaller $N_{\text {cut }}$ is, the more various topics are contained in the Web video groups. On the other hand, the larger $N_{c u t}$ is, the more closely related Web videos are contained in the Web video groups. Then users select Web video groups associated with the desired contents according to the hierarchical structure. Furthermore, users retrieve the desired Web videos from the selected Web video group based on the criterion $R_{N_{\text {cut }}}^{q}(i)$. In this way, even if users cannot write suitable queries that identify the desired contents, the hierarchical structure can navigate users to the desired contents.

\section{Experimental Results}

In this section, the effectiveness of the proposed Web video retrieval method is verified. 
Table 1 Details of datasets: $K$ is the total number of keywords that appear in text attached to Web videos, $N_{g}$ and $N_{m}$ are the parameters for constructing the subsets. To compute visual feature vectors, $p$ and $P_{v}$ were set to $48(H=12, S=2, V=2)$ and 1 , respectively. To extract textual features, we used title, description and comments attached to Web videos.

\begin{tabular}{|c|c|c|c|c|c|c|}
\hline & $\begin{array}{c}\text { Query } \\
\text { keyword }\end{array}$ & $\begin{array}{c}\text { Num. of } \\
\text { Web videos } \\
\text { in the dataset }\end{array}$ & $K$ & $N_{g}$ & $N_{m}$ & $\begin{array}{c}\text { Num. of } \\
\text { Web videos } \\
\text { in the subset }\end{array}$ \\
\hline Dataset 1 & apple & 3037 & 76498 & 15 & 70 & 775 \\
\hline Dataset 2 & galaxy & 3029 & 87778 & 15 & 70 & 661 \\
\hline Dataset 3 & jaguar & 3043 & 88396 & 15 & 70 & 574 \\
\hline Dataset 4 & sightseeing & 3044 & 93648 & 15 & 70 & 762 \\
\hline Dataset 5 & match & 1064 & 31022 & 15 & 70 & 487 \\
\hline Dataset 6 & game & 1053 & 27588 & 15 & 70 & 649 \\
\hline Dataset 7 & race & 1049 & 33670 & 15 & 70 & 556 \\
\hline Dataset 8 & Hokkaido & 1053 & 35903 & 15 & 70 & 521 \\
\hline Dataset 9 & $\begin{array}{c}\text { music } \\
\text { (in Japanese) }\end{array}$ & 1037 & 30173 & 15 & 70 & 586 \\
\hline Dataset 10 & $\begin{array}{c}\text { comedy } \\
\text { (in Japanese) }\end{array}$ & 1068 & 19742 & 15 & 70 & 529 \\
\hline
\end{tabular}

Table $2 N_{c u t}^{o p t}$ and maximum value of $Q_{N_{c u t}}$ for each subset.

\begin{tabular}{|c|c|c|}
\hline & $N_{\text {cut }}^{\text {opt }}$ & Maximum value of $Q_{N_{\text {cut }}}$ \\
\hline Subset 1 & 499 & 0.789 \\
\hline Subset 2 & 107 & 0.674 \\
\hline Subset 3 & 131 & 0.706 \\
\hline Subset 4 & 53 & 0.836 \\
\hline Subset 5 & 96 & 0.777 \\
\hline Subset 6 & 5 & 0.826 \\
\hline Subset 7 & 15 & 0.774 \\
\hline Subset 8 & 125 & 0.768 \\
\hline Subset 9 & 313 & 0.779 \\
\hline Subset 10 & 594 & 0.729 \\
\hline
\end{tabular}

\subsection{Datasets}

In the proposed method, metadata "related videos" on each Web video plays an important role to extract hierarchical structure for Web video retrieval. However, standard video retrieval datasets do not have the metadata. Therefore, we constructed datasets by our own for performing a fair comparison as follows. First, by using YouTube ${ }^{2}$, a keyword was given as a query, and the top 50 Web videos were obtained. Then we repeatedly obtained 10 Web videos contained in links, i.e., "related videos" 5 of the selected Web videos, and each dataset was constructed. Here, we collected only Web videos with lengths of less than 1800 seconds. Table 1 shows the detailed conditions of each dataset.

\subsection{Evaluation}

From each dataset, subsets were extracted according to Sec. 3 (Phase I). Next, from each subset, we extracted the parent Web video groups and the child Web video groups according to Sec. 4 (Phase II). In Table 2, $N_{c u t}^{\text {opt }}$ and the maximum

${ }^{5}$ In the experiment, we used YouTube Data API v2 to obtain the links, "related videos". 


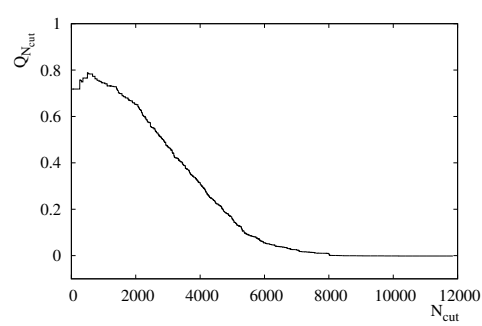

(a) Result for subset 1 .

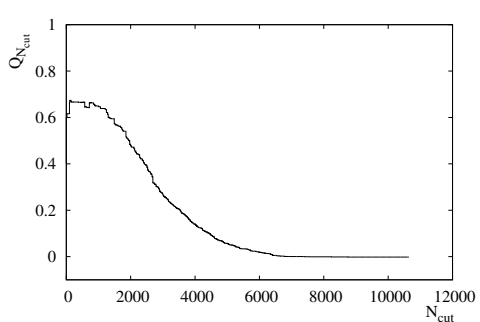

(b) Result for subset 2 .

Fig. 4 Change in modularity $Q_{N_{c u t}}$ to the number of cut edges, $N_{c u t}$.

Table 3 Relevant Web videos used in the experiment.

\begin{tabular}{|c|c|}
\hline & Relevant Web videos \\
\hline Dataset 1 & Web videos about \\
"Product of Apple Inc., iPhone"
\end{tabular}

value of $Q_{N_{c u t}}$ of each subset are shown, and Fig. 4 shows the change in modularity $Q_{N_{\text {cut }}}$ to $N_{\text {cut }}$ for subsets 1 and 2. Figure 5 shows the hierarchical structure of the largest parent Web video groups of subsets 1 and 2. This figure shows that the more closely related Web videos are obtained with an increase in the number of cut edges, $N_{c u t}$. Thus, it can be seen that the hierarchical structure of Web video groups can be estimated by our method.

Next, we quantitatively verify the effectiveness of the proposed Web video retrieval method shown in Sec. 5 (Phase III). We used recall, precision and average precision $(\mathrm{AP} @ k)$ defined as follows:

$$
\begin{aligned}
\text { Recall } & =\frac{\text { Num. of correctly retrieved Web videos }}{\text { Num. of relevant Web videos }}, \\
\text { Precision } & =\frac{\text { Num. of correctly retrieved Web videos }}{\text { Num. of retrieved Web videos }}, \\
\mathrm{AP} @ k & =\frac{1}{R_{k}} \sum_{i=1}^{k} x_{i} \text { prec }_{i},
\end{aligned}
$$

where $k$ is the number of Web videos provided as the retrieval results, $R_{k}$ is the number of "relevant Web videos" within $k$ Web videos of the retrieval results, $x_{i}$ is 1 if the $i$-th retrieved Web videos are "relevant Web videos" and 0 otherwise, and prec $_{i}$ is the precision when $i$ Web videos are retrieved. Table 3 shows relevant Web 


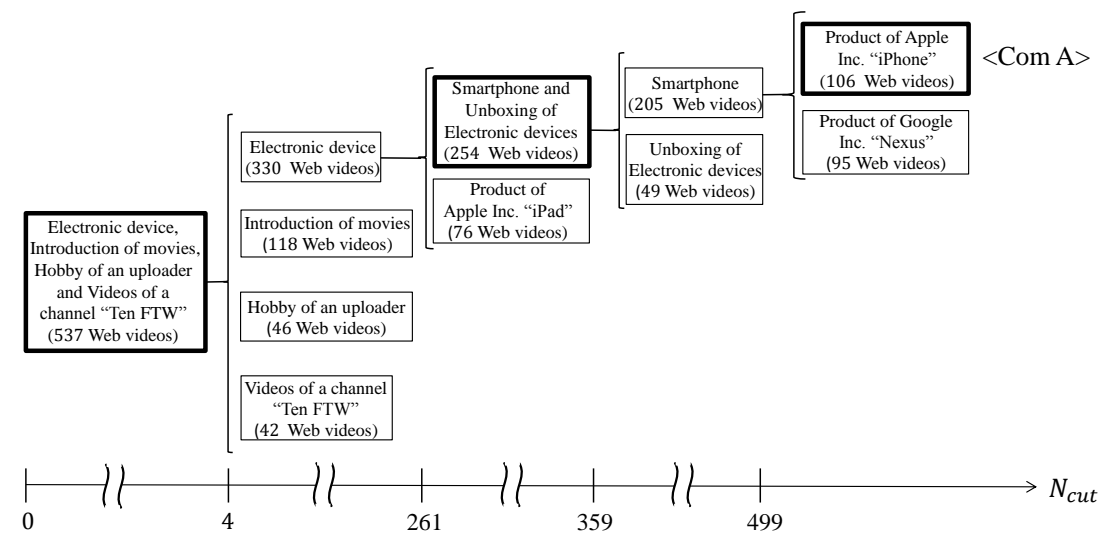

(a) Hierarchical structure of the largest parent Web video group of subset 1.

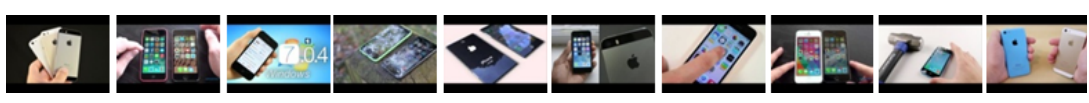

(b) Thumbnails of the top 10 retrieved Web videos in the order of left-to-right when "Com A" was selected.

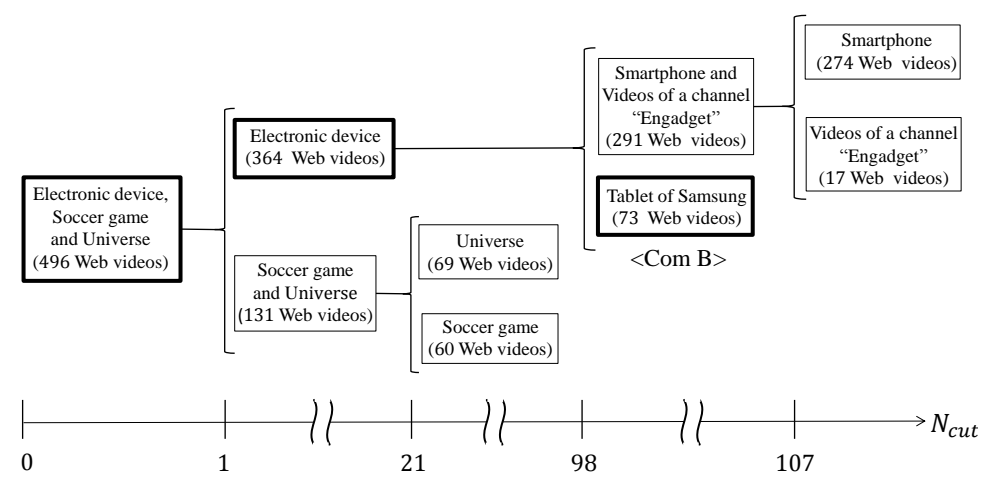

(c) Hierarchical structure of the largest parent Web video group of subset 2.

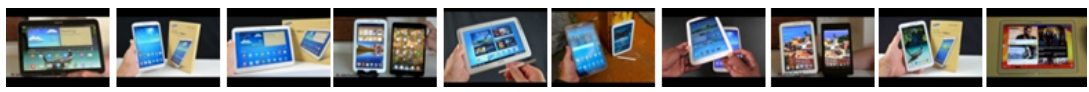

(d) Thumbnails of the top 10 retrieved Web videos in the order of left-to-right when "Com B" was selected.

Fig. 5 Hierarchical structure of Web video groups obtained by the proposed method. Squares with thick lines show Web video groups used in calculating recall and precision in Fig. 6 . We show only Web video groups containing more than 10 Web videos.

videos used in this experiment. We compare the retrieval results of the proposed method with the following reference methods.

Reference method (i)

This is a method that extracts hierarchical structure of Web video groups by using only link relationships between Web videos without the use of Web video features. 
Reference method (ii)

This is a conventional method [16] that extracts Web video groups by using link relationships between Web videos and the Web video features. This method does not extract the hierarchical structure.

Reference method (iii)

This is a method based on [17] with a Web video group extraction scheme by affinity propagation [8]. This method uses only Web video similarities without the use of link relationships between Web videos, and does not extract the hierarchical structure. In the experiment, we did not use the original similarities presented by [17] but utilized our proposed similarities defined in Eq. (1). For the evaluation, we ranked Web videos within Web video groups obtained by this method in descending order of the sum of the above similarities.

Reference method (iv)

This is a method based on clustering via latent semantic indexing (LSI) that uses only textual features of Web videos [18], which does not exhibit hierarchical structure to users. Note that an original method in [18] utilizes information of YouTube Playlists; however, we used TF-IDF vectors obtained in the same manner as our method in this experiment. Moreover, the number of dimensions of TF-IDF vectors after LSI were set to 200 as in a paper [18]. Each Web video is ranked on the basis of the attribution degree to the belonging cluster.

Reference method (v)

This is a method constructed by replacing our CCA-based video features by Non-negative matrix factorization (NMF)-based ones [11]. A paper [11] claims that coefficient vectors obtained by applying NMF to textual features are effective for building a latent semantic image representation. In this experiment, we used textual feature vectors $\boldsymbol{t}_{i}(i=1,2, \cdots, N)$ defined in Sec. 3.1. Note that we added constant values to all elements of the textual feature vectors to keep them non-negative. Then we implemented NMF by using the obtained vectors and extracted hierarchical structure of Web video groups in the same manner as our method. Note that we set the number of dimensions of vectors after performing NMF to 1500 and 500 for datasets 1-4 and datasets 5-10, respectively.

For all methods, we selected a Web video group that includes relevant Web videos the most, and Web videos were retrieved according to the ranking of Web videos. Then recall, precision and AP@ $k$ were calculated. Figure 6 shows recall and precision calculated for datasets 1 and 2. Note that several results per dataset are shown since we calculated recall and precision at several hierarchies in the proposed method and reference methods (i) and (v). As for the proposed method and reference methods (i) and (v), the larger $N_{c u t}$ is, the higher the precision is. This means that closely related Web videos are obtained by dividing the parent Web video group into their child Web video groups.

Moreover, Table 4 and Fig. 7 respectively show AP@ $k$ and the precision-recall curves for all datasets. From these results, we can see that our method has better performance than all reference methods for most datasets. Specifically, the effectiveness of using the heterogeneous video features can be seen by comparing our method with reference methods (i), (iv) and (v). When comparing our method with reference methods (ii), (iii) and (iv), we can see that it becomes feasible to accurately retrieve the desired Web videos by using the hierarchical structure of 

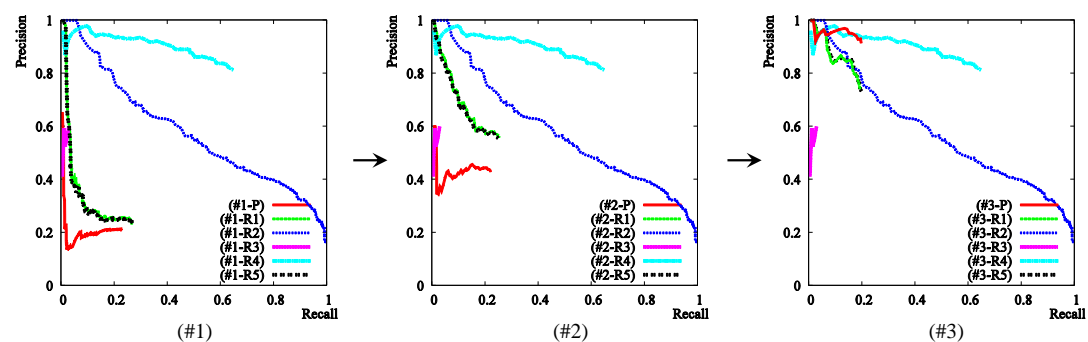

(a) Results for dataset 1: (\#1-P), (\#2-P), (\#3-P) are results of the proposed method where $N_{\text {cut }}$ are 0,265 and 500, respectively. (\#1-R1), (\#2-R1), (\#3-R1) are results of reference method (i) where $N_{\text {cut }}$ are 0,265 and 500, respectively. (R2), (R3) and (R4) show results of reference methods (ii), (iii) and (iv). (\#1-R5), (\#2-R5), (\#3-R5) are results of reference method (v) where $N_{\text {cut }}$ are 0,265 and 500, respectively.

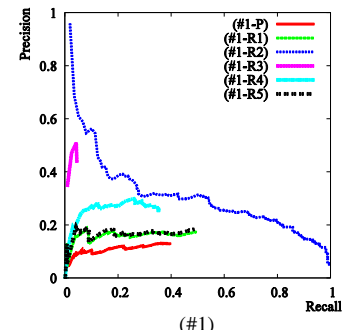

(\#1)

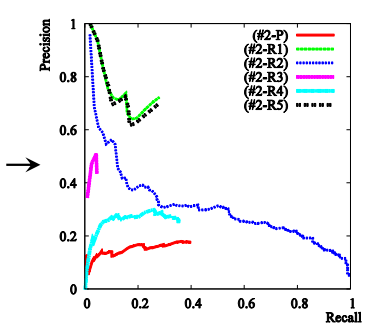

$(\# 2)$

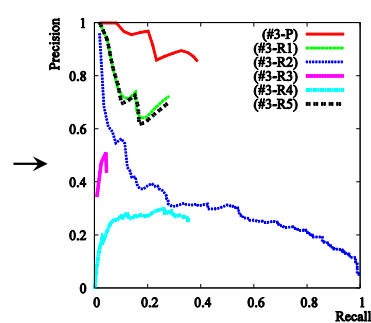

(\#3)

(b) Results for dataset 2: (\#1-P), (\#2-P), (\#3-P) are results of the proposed method where $N_{c u t}$ is 0,50 and 100 , respectively. (\#1-R1), (\#2-R1), (\#3-R1) are results of reference method (i) where $N_{\text {cut }}$ is 0,50 and 100 , respectively. (R2), (R3) and (R4) show results of reference methods (ii), (iii) and (iv). (\#1-R5), (\#2-R5), (\#3-R5) are results of reference method (v) where $N_{\text {cut }}$ is 0,50 and 100 , respectively.

Fig. 6 Precision-recall curves.

Table 4 Average precision (AP@k): For the proposed method and reference methods (i) and (v), the results where $N_{\text {cut }}$ are $N_{c u t}^{o p t}$ are shown. We defined the value of $k$ as the number of Web videos in each Web video group used for retrieval. Maximum values in each row are highlighted with asterisks.

\begin{tabular}{|c|c|c|c|c|c|c|}
\hline & $\begin{array}{c}\text { Proposed } \\
\text { method }\end{array}$ & $\begin{array}{c}\text { Reference } \\
\text { method } \\
\text { (i) }\end{array}$ & $\begin{array}{c}\text { Reference } \\
\text { method } \\
\text { (ii) }\end{array}$ & $\begin{array}{c}\text { Reference } \\
\text { method } \\
\text { (iii) }\end{array}$ & $\begin{array}{c}\text { Reference } \\
\text { method } \\
\text { (iv) }\end{array}$ & $\begin{array}{c}\text { Reference } \\
\text { method } \\
\text { (v) }\end{array}$ \\
\hline Dataset 1 & $0.958^{*}$ & 0.884 & 0.580 & 0.580 & 0.911 & 0.885 \\
\hline Dataset 2 & $0.937^{*}$ & 0.767 & 0.321 & 0.512 & 0.265 & 0.760 \\
\hline Dataset 3 & $1.00^{*}$ & 0.996 & 0.462 & 0.406 & 0.163 & 0.996 \\
\hline Dataset 4 & $0.993^{*}$ & $0.993^{*}$ & 0.793 & 0.905 & 0.970 & $0.993^{*}$ \\
\hline Dataset 5 & $0.906^{*}$ & 0.864 & 0.751 & 0.817 & 0.816 & 0.860 \\
\hline Dataset 6 & 0.962 & 0.945 & 0.947 & $0.992^{*}$ & 0.692 & 0.946 \\
\hline Dataset 7 & 0.948 & 0.991 & 0.893 & 0.929 & $0.999^{*}$ & 0.964 \\
\hline Dataset 8 & 0.944 & 0.935 & 0.590 & 0.812 & $0.956^{*}$ & 0.936 \\
\hline Dataset 9 & 0.999 & $1.00^{*}$ & 0.842 & 0.738 & $1.00^{*}$ & $1.00^{*}$ \\
\hline Dataset 10 & 0.998 & $1.00^{*}$ & 0.934 & 0.474 & 0.162 & $1.00^{*}$ \\
\hline
\end{tabular}

Web video groups. In particular, accurate retrieval can be realized since Web video groups are refined into more similar topics according to the hierarchical structure. From the results by our method and reference methods (iii) and (iv), it can be con- 


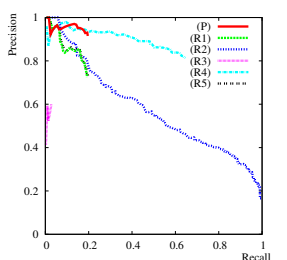

(a) Result for dataset 1 .

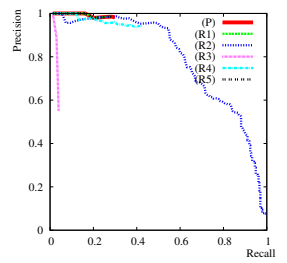

(d) Result for dataset 4 .

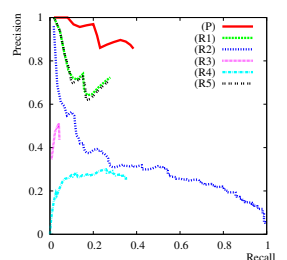

(b) Result for dataset 2 .

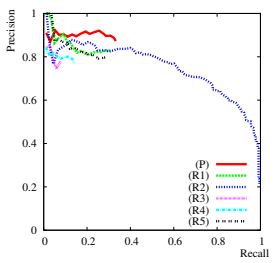

(e) Result for dataset 5 .

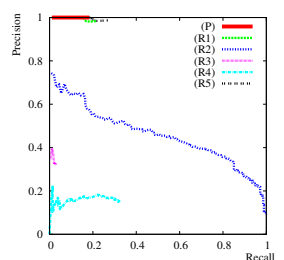

(c) Result for dataset 3 .

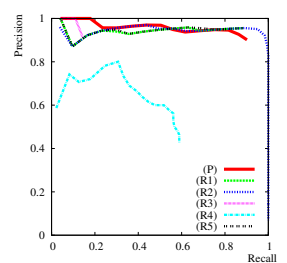

(f) Result for dataset 6 .

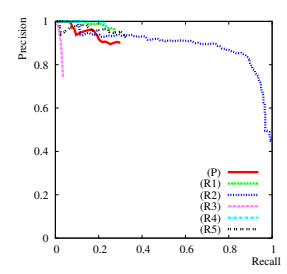

(g) Result for dataset 7 .

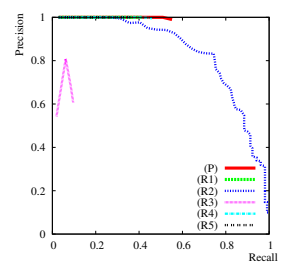

(i) Result for dataset 9 .

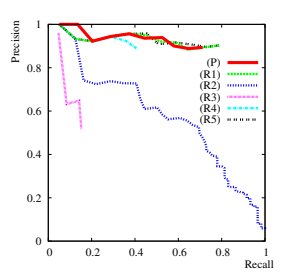

(h) Result for dataset 8 .

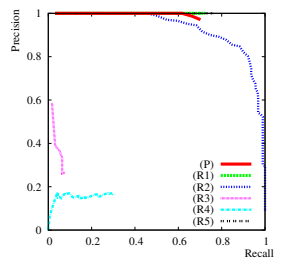

(j) Result for dataset 10 .

Fig. 7 Precision-recall curves: (P), (R1) and (R5) respectively correspond to our method and reference methods (i) and (v), where $N_{c u t}$ are $N_{c u t}^{\text {opt }}$. (R2), (R3) and (R4) correspond to reference methods (ii), (iii) and (iv), respectively. Note that reference method (ii) has higher recall than other methods since only this method is not based on hard clustering and all Web videos can belong to every Web video group.However, we consider our method that has higher precision is significant since it is desirable that users can retrieve the desired contents by browsing a small number of retrieval results.

firmed that link relationships between Web videos are useful for obtaining similar Web videos accurately whereas the only use of the video features is insufficient. On the other hand, precision (Fig. 7) of our method is less than reference methods (i), (ii), (iv) and (v) for dataset 7 . We guess this is because those relevant Web videos can be simply expressed by using only link relationships or textual features without the use of heterogeneous video features. To solve such a problem, in the future, we need to develop new schemes to select the effective features for retrieval of the desired contents. 


\section{Limitation and Future Work}

In this section, we discuss limitation and future work of this paper.

\subsection{Discussion on a User Interface}

In this paper, we name each Web video group in the hierarchies shown in Fig. 5 by checking contents in Web video groups manually. In the future, we should develop a scheme for automatically naming the hierarchies. We consider it is necessary to reveal salient keywords in each Web video group and estimate hierarchical relationships between the keywords [4,20].

Moreover, we explain how navigation is presented to a user for Web video retrieval. In Fig. 8, we show an example of a user interface constructed on the basis of a network drawing algorithm [15]. As shown in this figure, for a given query, we exhibit hierarchical structure of Web video groups to a user via the interface. When a user selects a Web video group, the user can grasp the overview of the selected Web video group via the visualized network and can retrieve each Web video in descending order of the rankings based on Eq. (8). In (b), (c) and (d) in Fig. 8, the left sides of each figure depict the networks visualized on the basis of an algorithm [15], and the right sides show rankings of each Web video in the selected Web video group. Then, until the desired contents are retrieved, a user repeatedly selects Web video groups in several hierarchical levels by browsing names of each Web video group.

In general, topics contained in the desired contents belong to various hierarchical levels according to each user. In our method, the exhibition of the hierarchical structure via a user interface enables navigation to the desired contents.

\subsection{Discussion on Computational Cost}

Here, we discuss computational cost of our method with regard a real-world deployment. In our method, computational cost of Web video similarities based on CCA is $O\left(N^{3}\right)$ and that of extracting the hierarchical structure is $O\left(M_{s}^{2} N_{s}\right)$, where $M_{s}$ and $N_{s}$ are the numbers of edges and nodes in the target subset, respectively [1,22]. Also, we need to recalculate them when a new query is given by a user and new Web videos are added or deleted. Since their recalculation should be avoided for a real-world deployment, we should develop schemes to efficiently update Web video similarities and the hierarchical structure. To update Web video similarities, incremental schemes will be useful [27]. On the other hand, to keep the hierarchical structure updated and select the best $N_{c u t}$, i.e., $N_{c u t}^{o p t}$, a dynamic network analysis algorithms [26] will be necessary. In the future, we should introduce them into our method for a real-world deployment. 


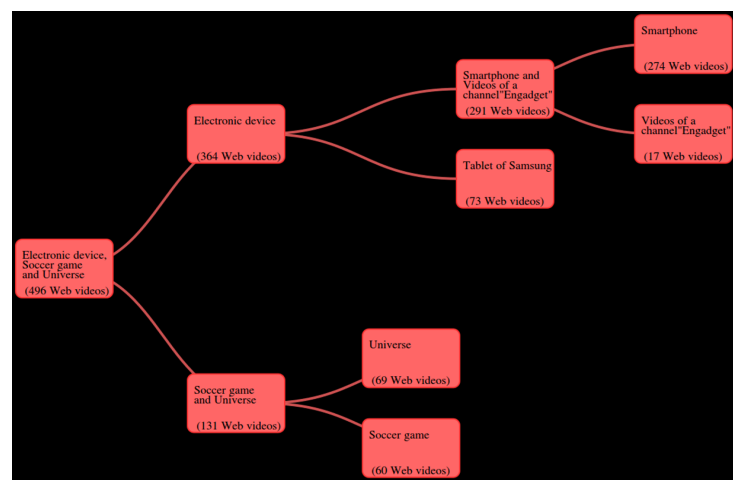

(a)

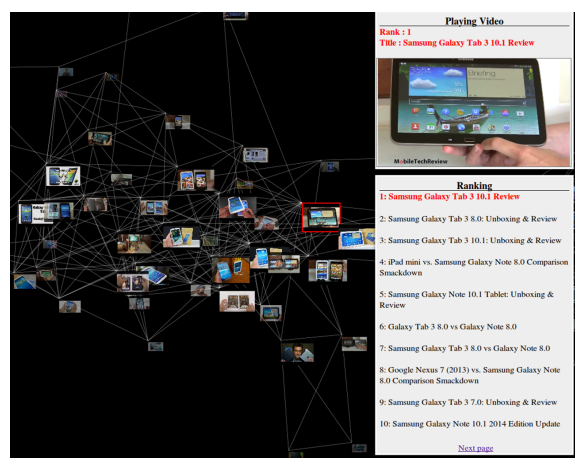

(b)

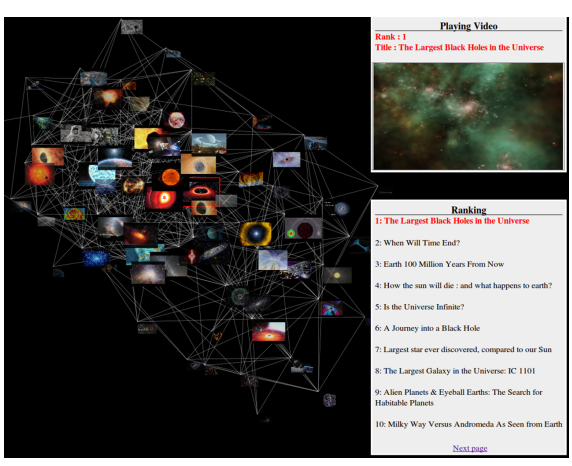

(c)

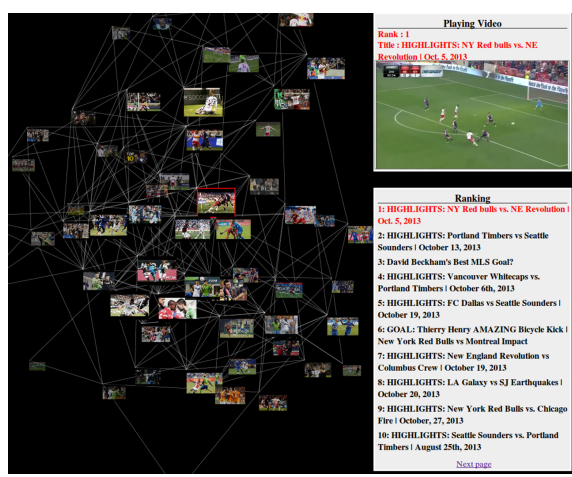

(d)

Fig. 8 Example of a user interface for dataset 2. This interface is built based on a network drawing algorithm [15]. (a): This interface exhibits the hierarchical structure for a given query "galaxy" to a user. (b), (c) and (d): This interface respectively exhibits details of Web video groups "Tablet of Samsung", "Universe" and "Soccer game" in Fig. 5 when a user selects them from the hierarchical structure.

\section{Conclusions}

In this paper, we have proposed a Web video retrieval method using hierarchical structure of Web video groups. The proposed method enabled retrieval of the 
desired Web videos even if users cannot write suitable queries that identify the desired contents by the two contributions, i.e., extraction of hierarchical structure of Web video groups, and Web video retrieval that uses the hierarchical structure. In the first contribution, the combination use of link relationships between Web videos and heterogeneous video features can overcome the limitation in the retrieval performance when only a single modality is used. In the second contribution, even if users cannot write suitable queries, the hierarchical structure enabled navigation to the desired contents. Experimental results have verified the effectiveness of the proposed Web video retrieval method. Future work includes developing a method for automatically naming each Web video group in the hierarchical structure. Also, in the future, we should develop schemes to efficiently update Web video similarities and the hierarchical structure when a new query is given and new Web videos are added or deleted.

Acknowledgements This work was partly supported by Grant-in-Aid for Scientific Research (B) 25280036, Japan Society for the Promotion of Science (JSPS), and Grant-in-Aid for Scientific Research on Innovative Areas 24120002 from the MEXT. We are grateful that a publisher, Springer permits us to deposit this accepted manuscript in the open access repository. The final publication is available at "http://link.springer.com/article/10.1007/s11042-015-2976-8".

\section{References}

1. Allaire, G., Kaber, S.M.: Numerical Linear Algebra. Springer-Verlag New York (2008)

2. Arenas, A., Duch, J., Fernandez, A., Gomez, S.: Size reduction of complex networks preserving modularity. New J. Phys. 9 (176), 604-632 (2007)

3. Astola, J., Haavisto, P., Neuvo, Y.: Vector median filters. In: Proc. IEEE, pp. 678-689 (1990)

4. Brachman, R.: What is-a is and isn't: An analysis of taxonomic links in semantic networks. IEEE Computer 16(10), 30-36 (1983)

5. Butafogo, R.A., Schneiderman, B.: Identifying aggregates in hypertext structures. In: Proc. 3rd ACM Conf. Hypertext, pp. 63-74 (1991)

6. Cheng, X., Dale, C., Liu, J.: Statistics and social network of youtube videos. In: Proc. IEEE Int. Workshop on Quality of Service, pp. 229-238 (2008)

7. Fan, J., Elmagarmid, A.K., X. Zhu, W.G.A., Wu, L.: Classview: Hierarchical video shot classification, indexing and accessing. IEEE Trans. Multimedia 6(1), 70-86 (2004)

8. Frey, B.J., Dueck, D.: Clustering by passing messages between data points. Science 315(5814), 972-976 (2007)

9. Gantz, J., Reinsel, D.: The digital universe in 2020: Big data, bigger digital shadows, and biggest growth in the far east. In: IDC iView (2012)

10. Gargi, U., Wenjun, L., Vahab, M., Sangho, Y.: Large-scale community detection on youtube for topic discovery and exploration. In: Proc. Int. AAAI Conf. Weblogs and Social Media, pp. 486-489 (2011)

11. González, F.A., Caicedo, J.C., Nasraoui, O., Ben-Abdallah, J.: Nmf-based multimodal image indexing for querying by visual example. In: Proc. ACM Int. Conf. Image and Video Retrieval, pp. 366-373 (2010)

12. Harakawa, R., Hatakeyama, Y., Ogawa, T., Haseyama, M.: An extraction method of hierarchical web communities for web video retrieval. In: Proc. IEEE Int. Conf. Image Processing, pp. 4397-4401 (2013)

13. Haseyama, M., Ogawa, T.: Trial realization of human-centered multimedia navigation for video retrieval. Int. Journal of Human-Computer Interaction 29(2), 96-109 (2013)

14. Haseyama, M., Ogawa, T., Yagi, N.: A review of video retrieval based on image and video semantic understanding. ITE Trans. MTA 1(1), 2-9 (2013)

15. Hatakeyama, Y., Haseyama, M.: An effective visualization method based on web community extraction using hyperlink between web videos and its application to retrieval. In: Proc. Int. Tech. Conf. Circuits/Systems, Computers and Communications, pp. 371-374 (2010) 
16. Hatakeyama, Y., Ogawa, T., Asamizu, S., Haseyama, M.: A novel video retrieval method based on web community extraction using features of video materials. IEICE Trans. Fundamentals E92-A(8), 1961-1969 (2009)

17. Hindle, A., Shao, J., Lin, D., Lu, J., Zhang, R.: Clustering web video search results based on integration of multiple features. World Wide Web 14(1), 53-73 (2011)

18. Kamie, M., Hashimoto, T., Kitagawa, H.: Effective web video clustering using playlist information. In: in Proc. ACM Symp. Applied Computing, pp. 949-956 (2012)

19. Kleinberg, J.M.: Authoritative sources in a hyperlinked environment. Jounal of ACM 46(5), 604-632 (1999)

20. Miller, G.A.: Wordnet: A lexical database for english. Commun. ACM 38(11), 39-41 (1995)

21. Nagasaka, A., Tanaka, Y.: Automatic video indexing and fullvideo search for object appearances. In: Proc. IFIP 2nd Working Conf. Visual Database Systems, pp. 113-127 (1991)

22. Newman, M.E.J., Girvan, M.: Finding and evaluating community structure in networks. Phys. Rev. E 69 (026113) (2004)

23. Ngo, C.W., Pong, T.C., Zhang, H.J.: On clustering and retrieval of video shots through temporal slices analysis. IEEE Trans. Multimedia 4(4), 446-458 (2002)

24. Nielsen, A.: Multiset canonical correlations analysis and multispectral, truly multitemporal remote sensing data. IEEE Trans. Image Processing 11(3), 293-305 (2002)

25. Nitanda, N., Haseyama, M.: Audio-based shot classification for audiovisual indexing using pca, mgd and fuzzy algorithm. IEICE Trans. Fundamentals E90-A(8), 1542-1548 (2007)

26. Papadopoulos, S., Kompatsiaris, Y., Vakali, A., Spyridonos, P.: Community detection in social media. Data Mining and Knowledge Discovery 24(3), 515-554 (2012)

27. Ross, D.A., Lim, J., Lin, R.S., Yang, M.H.: Incremental learning for robust visual tracking. Int. J. Comput. Vis. 77(1-3), 125-141 (2008)

28. Sang, J., Xu, C.: Browse by chunks: Topic mining and organizing on web-scale social media. ACM Trans. Multimedia Comput. Commun. Appl. 7S(1), 30:1-30:18 (2011)

29. Sebastiani, F.: Machine learning in automated text categorization. ACM Computing Surveys $\mathbf{3 4}(1), 1-47(2002)$

30. Taskiran, C., Chen, J.Y., Albiol, A., Torres, L., Bouman, C.A., Delp, E.J.: Vibe: A compressed video database structured for active browsing and search. IEEE Trans. Multimedia 6(1), 103-118 (2004)

31. Wang, Y., Belkhatir, M., Tahayna, B.: Near-duplicate video retrieval based on clustering by multiple sequence alignment. In: Proc. ACM Int. Conf. Multimedia, pp. 941-944 (2012)

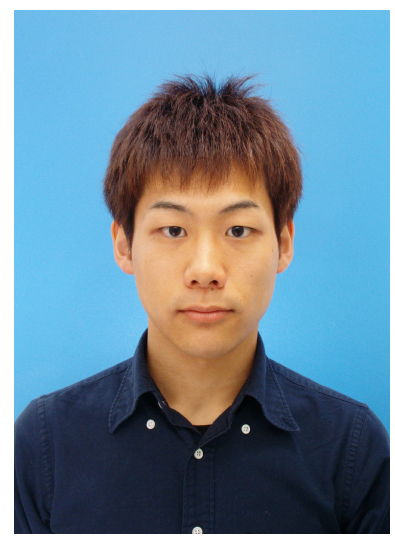

Ryosuke Harakawa received his B.S. and M.S. degrees in Electronics and Information Engineering from Hokkaido University, Japan in 2013 and 2015, respectively. He is currently pursuing a Ph.D. degree at the Graduate School of Information Science and Technology, Hokkaido University. His research interests include audiovisual processing and Web mining. He is a student member of the IEEE, IEICE, and Institute of Image Information and Television Engineers (ITE). 

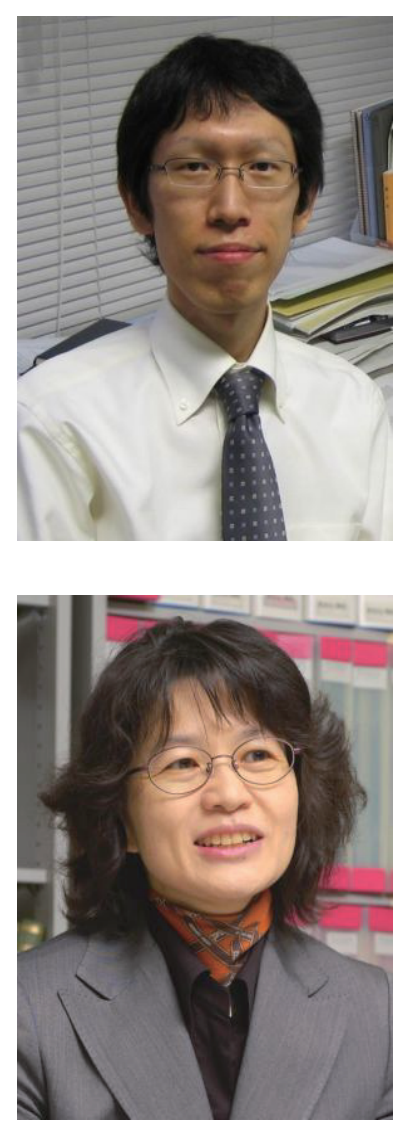

of Japan (ASJ).
Takahiro Ogawa received his B.S., M.S. and Ph.D. degrees in Electronics and Information Engineering from Hokkaido University, Japan in 2003, 2005 and 2007, respectively. He is currently an assistant professor in the Graduate School of Information Science and Technology, Hokkaido University. His research interests are multimedia signal processing and its applications. He has been an Associate Editor of ITE Transactions on Media Technology and Applications. He is a member of the IEEE, EURASIP, IEICE, and Institute of Image Information and Television Engineers (ITE).

Miki Haseyama received her B.S., M.S. and Ph.D. degrees in Electronics from Hokkaido University, Japan in 1986, 1988 and 1993, respectively. She joined the Graduate School of Information Science and Technology, Hokkaido University as an associate professor in 1994. She was a visiting associate professor of Washington University, USA from 1995 to 1996. She is currently a professor in the Graduate School of Information Science and Technology, Hokkaido University. Her research interests include image and video processing and its development into semantic analysis. She has been a Vice-President of the Institute of Image Information and Television Engineers, Japan (ITE), an Editor-in-Chief of ITE Transactions on Media Technology and Applications, a Director, International Coordination and Publicity of The Institute of Electronics, Information and Communication Engineers (IEICE). She is a member of the IEEE, IEICE, Institute of Image Information and Television Engineers (ITE) and Acoustical Society 